
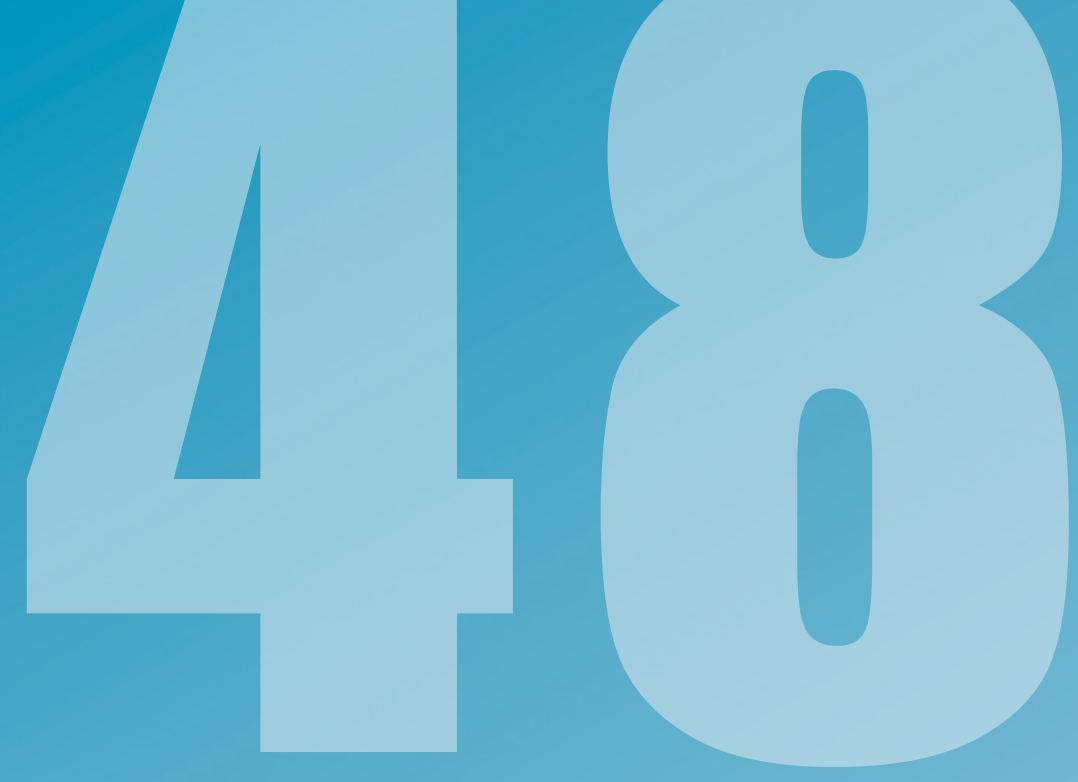

ÁMBITOS

REVISTA

INTERNACIONAL

\title{
DE COMUNICACIÓN
}

$N^{\circ} 48$

EDICIÓN PRIMAVERA

2020

ISSN: 1139-1979

E-ISSN: 1988-5733

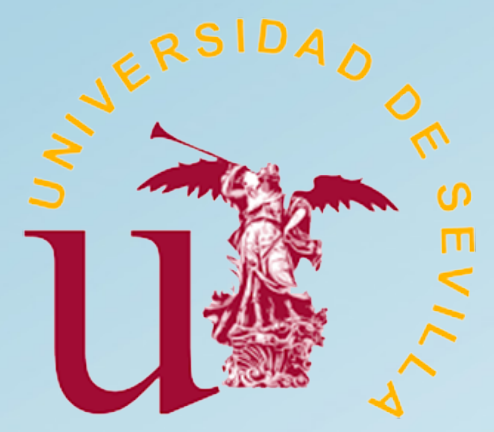




\section{ÍNDICE}

MONOGRAFICOS MONOGRAPHS

Presentación Monográfico. Investigación y comunicación en las organizaciones

José Luis Rojas Torrijos-Francisco Javier Paniagua Rojano

Automatizaciones en la gestión de la comunicación en las instituciones públicas

Automations in communication management in public institutions

Alejandro Álvarez-Nobell, Antonio Castillo-Esparcia, Isabel Ruiz-Mora

Futbolistas en Instagram: análisis del marketing de influencia realizado por los capitanes de Primera División en España

Footballers on Instagram: influence marketing analysis by Spain First Division captains

Jesús Segarra-Saavedra, Tatiana Hidalgo-Marí

Comunicación interna, compromiso y bienestar de la plantilla: el caso de Admiral Seguros

Internal communication, commitment and well-being of the workforce: the case of Admiral Seguros

Andrea Castro-Martínez, Aimiris Sosa Valcarcel, Emelina Galarza Fernández

$56-78$

Estrategia y comunicación en redes sociales: Un estudio sobre la influencia del movimiento RealFooding

Strategy and communication in social media: A study about the influence of the RealFooding movement

Cristina González Oñate, Adela Martínez Sánchez

Estudio de la presencia digital en MotoGP: Estudio de caso Jorge Lorenzo en Instagram Study of the digital presence in MotoGP: Jorge Lorenzo case study in Instagram 
El uso del color en la construcción de comunicación eficaz para cartelería.

\section{Estudio de caso: actividades formativas de emprendimiento}

The use of color in the construction of effective communication for posters.

A case study: training and entrepreneurship activities

Alberto Luis García García, Clara DePedro-Garabito, Maciej Wysokinski

Redes sociales, convergencia y narrativas transmedia en la promoción de las Islas Canarias

Social networks, convergence and transmedia narratives in the promotion of the Canary Islands

Noelia Iñesta Fernández, José Sixto García

Aproximación al estudio de la estrategia de comunicación de las universidades andaluzas en Linkedln

Approach to the study of the communication strategy of Andalusian universities on Linkedln

Estefanía Cestino González

Evolución de las estrategias de patrocinio en los esports en España: 2013-2021

Evolution of sponsoring strategies in esports in Spain: 2013-2021

F. J. Cristòfol, Álvaro Martínez-Ruiz, Ignacio Román-Navas, Carmen Cristófol-Rodríguez

188-204

El emplazamiento inverso como estrategia de comunicación corporativa para

HBO: el caso de True Blood

The reverse product placement as a corporate communication strategy for HBO: The True Blood case

Víctor Álvarez Rodríguez

Análisis del brand placement en La casa de papel

Analysis of brand placement in "Money heist"

Araceli Castelló-Martínez

\section{RESEÑAS REVIEWS}

\section{Necesaria aproximación global a la televisión en Europa}

Necessary global approach to the television in Europe

Cristina Zapatero Flórez

246-249 
El conflicto vasco a través de las producciones televisivas

The basque conflict through television productions

Pablo Berdón-Prieto

250-253

Transmutación de la comunicación en la Sociedad Red: retos y oportunidades

Transmutation of communication in network society: challenges and opportunities

Lucia Ballesteros-Aguayo

254-259 


\section{Evolución de las estrategias de patrocinio en los esports en España: 2013-2021}

Evolution of sponsoring strategies in esports in

Spain: 2013-2021

F. J. Cristòfol, ESIC, Business \& Marketing School, C/ Severo Ochoa, 49, 29590, Málaga

Fjcristofol@esic.edu|https://orcid.org/0000-0002-0967-3514

Álvaro Martínez-Ruiz, ESIC, Business \& Marketing School, C/Severo Ochoa, 49, 29590, Málaga

322218@students.esic.edu | https://orcid.org/0000-0002-5237-4659

Ignacio Román-Navas, ESIC, Business \& Marketing School, C/ Severo Ochoa, 49, 29590, Málaga

321832@students.esic.edu | https://orcid.org/0000-0003-1417-8515

Carmen Cristófol-Rodríguez, Universidad de Málaga.

C/ León Tolstoi s/n, 29010, Málaga

carcrir@uma.es | https://orcid.org/0000-0001-9766-6500

DOI: https://dx.doi.org/10.12795/Ambitos.2020.i48.10 


\section{Resumen}

En este artículo se expone qué son los esports y la evolución de su industria en cifras durante los últimos cinco años. Se analizan tanto los resultados económicos como datos de audiencia a nivel internacional y se analiza el desarrollo del patrocinio durante este mismo periodo en España. Con las cifras obtenidas de estudios secundarios y la opinión de expertos, tratamos de extrapolar la evolución esperada para 2021. Debido al gran desconocimiento general sobre el sector y sus opciones publicitarias, se expone cómo se desarrolla el patrocinio en esta industria, así como su potencial evolutivo. Para todo ello, se utiliza una metodología de análisis y clasificación de datos desde 2013 hasta 2019. También se producen estimaciones de cifras y la evolución del sector hasta 2021 basadas en el crecimiento anterior, fuentes secundarias especializadas como son Newzoo y Antevenio; y entrevistas a cinco profesionales de los diferentes agentes del ecosistema de los deportes electrónicos: un social media, un redactor, un responsable de contenidos, un lobbyist y un jefe de sección. Se describe a lo largo de la investigación las diferentes acciones llevadas a cabo en España por los patrocinadores principales. Se demuestra que el patrocinio en los esports evoluciona de forma positiva a un ritmo exponencial de forma paralela a la industria que concluye en que el crecimiento de los deportes electrónicos conllevará la consolidación y el crecimiento del patrocinio en la industria.

\section{Abstract}

This article describes what esports are and the development based on figured of the related industry in the last five years. At international level are analysed both economic results and audience data and for the Spanish market is evaluated the sponsorship development. Based on the data from secondary sources and the valuation of experts, this assessment tries to extrapolate the expected evolution by 2021. Due to a general lack of knowledge about the sector and its advertising options, it is exposed how sponsorship has developed in this industry as well as its potential growth. For this purpose, a methodology of analysis and classification of data from 2013 to 2019 has been used. Estimations of figures and the evolution of the sector up to 2021 are also produced based on the previous growth, specialized secondary sources such as Anwo and Antevenio; and interviews with five professionals from the different agents of the esports industry: a social media, an editor, a content manager, a lobbyist and a managerial staff. The different actions carried out in Spain by the main sponsors are described throughout this investigation. It is shown that sponsorship in esports raises at an exponential rate in parallel to the industry that concludes that the growth of esports will entail the consolidation and development of sponsorship in the industry 
Palabras clave: esports, Marketing digital, Industria emergente, Videojuegos, Deportes electrónicos

Keywords: esports, Digital Marketing, Emerging Industry, Video Games

\section{INTRODUCCIÓN}

Los esports se han convertido en un fenómeno mundialmente conocido y actualmente ya hay torneos de millones de dólares en premios y patrocinadores, programas de online y de televisión exclusivos y todo tipo de eventos relacionados con los deportes electrónicos competitivos.

Bien es cierto que los esports están basados en la premisa de que ocurren gracias a los videojuegos, pero la industria del videojuego es muy diferente a la de los esports: esta primera está muy explorada, mientras que la de los esports, es una industria aún en crecimiento y desarrollo.

El objetivo de la presente investigación es recopilar información ya existente sobre los esports desde 2013 hasta la actualidad recogiendo predicciones de analistas de datos y de profesionales del sector para analizar el futuro de esta industria de cara a los próximos años.

\section{MARCO TEÓRICO}

La definición de los esports o deportes electrónicos y la forma de referirse a ellos aún es confusa para muchas personas, incluso para los expertos. Existe una definición bastante aceptada en el diccionario de Cambridge (2018), que dice que es "la actividad basada en jugar a juegos de ordenador contra otras personas en Internet, a menudo por dinero, ya sea con público mediante Internet, o en eventos especiales organizados". Por otro lado, aún hay discusión por la terminología usada para referirse a esta actividad pues vemos el término escrito como esports, eSports o e-sports, aunque generalmente la más reconocida es 'esports' de forma internacional, deportes electrónicos en español.

Según afirma Chris Baker (2016), reportero de la revista Rolling Stone, los inicios de los esports datan de 1972, concretamente en la universidad de Stanford, donde se disputó un torneo de Spacewar, el primer torneo de videojuegos de la historia. Participaron cerca de treinta personas, y el primer premio fue una suscripción anual a la revista Rolling Stone.

A pesar de este anecdótico "Primer torneo de esports de la historia", no es hasta 1980 cuando se da el primer torneo serio y con un número consistente de participantes. 'The 'Space Invaders Championship' fue el primer torneo de esports notorio, organizado 
directamente por la compañía del juego, Atari. El torneo recibió una notable recepción por el público reuniendo a 10.000 participantes (Bountie, 2018).

Sin embargo, no fue hasta los 90 cuando los esports empezarían a alcanzar cierta fluidez y popularidad, gracias a la iniciativa de varias compañías desarrolladoras que promocionaban torneos de sus propios videojuegos, como la QuakeCon (Wagner, 2006) y el 'Nintendo World Champions' (AtariHQ, 1997); y gracias también a las recién llegadas organizaciones de videojuegos, dedicadas exclusivamente a los esports como disciplina: la 'Profesional Gamers League' (G Synnaeve, \&P Bessiere, 2012) y la 'Cyberathlete Professional League' (Wagner, 2006), potenciadas principalmente por el juego competitivo estrella de los 90, 'Starcraft' (Lazzari, 2017).

Iniciada la década de los 2000 , se produce un hecho de gran relevancia en la historia de los esports: el nacimiento de la que muchos denominan actual Meca de los esports (Mediavilla, 2018). Hablamos del nacimiento de Kespa (Korea esports Association), organización lanzada en el 2000 por el gobierno coreano gracias a la iniciativa del ministro de cultura, deportes y turismo de aquel entonces (Lazzari, 2017).

Durante esta década toman valor las competiciones de esports gracias al lanzamiento de varios videojuegos que serían claves para estratificar los diferentes estilos en géneros de deportes electrónicos.

El lanzamiento de 'Counter Strike' cambió por completo el panorama de los shooter en los esports, que apenas se habían visto involucrados, con Quake. Counter Strike se profesionalizó a niveles exponenciales, propiciando la creación de la anteriormente mencionada CPL (CyberAthlete Pro-League) y de la CGS (Championship Gaming Series) (Ferguson Mitchell, 2018).

World Of Warcraft fue otro de esos títulos decisivos para la década de los 2000. Trajo consigo una fuerte inversión de Blizzard (empresa creadora del juego) en torneos del propio título, potenciando también el otro juego anteriormente mencionado de Blizzard, Starcraft.

Lo realmente interesante de Starcraft, un juego del género RTS (Real Time Strategy), fue todo lo que se creó a partir de éste, gracias a los mods de mapas añadidos en el juego. Hablamos del nacimiento de uno de los géneros referentes en los esports, los MOBA (Multiplayer Online Battle Arena), siendo el primer MOBA íntegramente un mod de creación de Starcraft, que daba opción a crear nuevos mapas, de los que saldrían posteriormente títulos absolutamente referentes como League Of Legends o Dota 2 lanzados en 2009 y 2013 respectivamente (Martín, 2017).

Entrado el 2013 los esports ya están consolidados a nivel global y aunque aún hay debate sobre si son considerados deporte o no, tienen un impacto muy importante que 
nadie puede negar. Este debate no sería zanjado hasta 2017 cuando el COI (Comité Olímpico Internacional), en un comunicado oficial, declaró a los esports como un deporte o actividad deportiva, debido a que los jugadores involucrados están preparados y entrenados con intensidad, al igual que los atletas tradicionales.

Para poder hablar de la evolución de las estrategias e inversión de marketing en el sector, se debe hablar primero de las cifras globales de los esports y la evolución a lo largo de estos cinco años. El año 2013 ya fue un año muy importante en la industria pues más de 71.500 .000 personas vieron competiciones, de los cuales 32.000 .000 fueron del videojuego League of Legends, donde algunos torneos como el de DOTA tenía un premio de más de 2.874 .380 dólares americanos (Warr, 2014). En total, la industria movió más de 25 millones de dólares en premios, obteniendo una cifra aproximada de 70 millones de dólares en movimientos. Sin duda unas cantidades ya muy importantes para una industria aún emergente que tenía todavía un futuro incierto. Sin embargo, los datos lo dejaban claro, los esports estaban en auge y no pararían ahí. Podemos ver como estas cifras sólo aumentaron en 2014 y futuros años.

En 2014, según el reporte anual (2016) de Newzoo, hubo más de 204 millones de espectadores globales tanto de entusiastas (90 millones) como de consumidores ocasionales (114 millones) esto fue un aumento muy significativo con respecto al año anterior pues fueron casi 130 millones de espectadores más. Respecto al dinero movido por la industria, fueron más de 194 millones de dólares en total durante el año.

El año 2015 tampoco fue una excepción en cuanto a crecimiento en el sector. En referencia a la audiencia, se obtuvo un notable aumento del $11,2 \%$ en relación con el año anterior, pasando de 204 millones de espectadores a 226 millones, con una importante rotación de público ocasional a público entusiasta. En cuanto a evolución de ingresos y capital en la industria, progresó en un 67\%, con un crecimiento de 131 millones de dólares (Newzoo, 2016).

El 2016 fue un punto de inflexión importante, ganando 56 millones de espectadores en apenas un año, lo que supuso un crecimiento de casi el $20 \%$. La inversión creció en un $34 \%$, pasando a facturar casi 500 millones de dólares. Por otro lado, 2017 tuvo un crecimiento bastante similar a su predecesor, del 33\% en inversión, y del 19,3\% en audiencia (Newzoo, 2018).

En el año 2018 las cifras de visitas totales fueron 380 millones a nivel global, de las cuales 165 millones son entusiastas y 215 millones son casuales (Newzoo, 2018). Por otro lado, también por parte de Newzoo, podemos ver que se trabajó con más de 906 millones de dólares durante todo el año, de los cuales 694 millones de dólares fueron exclusivamente de inversiones de marcas en el sector.

Aún no se ha cerrado el 2019 pero la mayoría de las competiciones han concluido y ya empiezan a aparecer los datos relativos a este año. Según Newzoo, en 2019 las cifras 
de visitas a nivel internacional son aproximadamente 454 millones, siendo 253 millones por personas espectadores casuales y 201 millones espectadores entusiastas del sector. En cuanto a los ingresos, se estima que la industria ha movido en total este año más de 1.000 millones de dólares, siendo la gran parte (879 millones) gracias a los patrocinadores que rodean las competiciones (Newzoo, 2019).

El principal retorno que tiene la inversión en esports, por parte de los clubes, son los sponsors. En sus inicios, pudimos observar que la industria apenas se movía por torneos organizados por los creadores del propio título, teniendo un retorno únicamente con los premios de los torneos, haciendo absolutamente imposible su continuidad y estabilidad al no tener solidez alguna en el tiempo ni el club ni el videojuego. Esto se asemeja mucho a la industria de los grandes deportes tradicionales donde el agente principal de su facturación se encuentra en los patrocinios de las marcas (Guiñón Moliner, 2017).

En la historia de los esports podemos percibir un cambio considerable de profesionalización cuando gracias a unos títulos ya consolidados, se empiezan a involucrar marcas que les dan continuidad y contenido a las competiciones. Andrew Meola nos menciona varios de los ejemplos de las empresas grandes involucradas en los esports (Meola, 2018):

- Intel: Empresa pionera en patrocinio de esports, tiene uno de los torneos más relevantes de la historia del sector: ESL Másters. Este es el torneo de esports que más tiempo lleva activo, uno de los que más inversión y premios mueve, y absoluto referente en títulos como Counter Strike, albergando sus competiciones de más nivel. Aparte de este torneo, Intel también patrocina a varios equipos de esports, entre los que se encuentran las organizaciones chinas Edward Gaming e Invictus Gaming.

- Coca-Cola: La multinacional de bebidas, entró en los esports en 2013 de la mano de Riot Games, convirtiéndose en el primer patrocinador oficial de lo que hoy en día es la competición de esports más seguida del mundo: el World Championship de Riot Games. Este contrato de patrocinador se prolongó hasta 2016, donde finalmente no fue renovado. Tras esto, Coca-Cola siguió patrocinando eventos de esports, creando su propia competición de FIFA: la eCopa Coca-Cola, de la mano de los desarrolladores del propio título EA Sports.

- Comcast Xfinity: Esta empresa subsidiaria de Comcast Corporation es un referente en servicios de telecomunicaciones por cable en Estados Unidos. Esta fue predecesora de todas las empresas de telecomunicaciones que han ido entrando en los esports, dándose cuenta de que tienen un potencial segmento de mercado con un claro público objetivo ya que para jugar o 
consumir esports se necesita una conexión rápida y estable. Entró en el 2016 y actualmente es patrocinador tanto de la ESL (como anteriormente vimos con Intel) como del equipo americano Evil Geniuses.

- Red Bull: La empresa austriaca de las bebidas energéticas es posiblemente la empresa mejor posicionada en esports del momento. Cómo relata Christian Kresse (2016): "Red Bull es el rey del marketing de contenidos en Esports". Interesándose por ellos desde sus inicios, acabó entrando en la industria en 2008. Desde entonces, han seguido una estrategia de marketing muy clara, con una serie de patrocinios y contenido que harían que el nombre de la marca calara en todos los seguidores de la disciplina. Su primer paso fue patrocinar eventos, creados y financiados por ellos mismos. Pese a ir poco a poco, hoy en día Red Bull cuenta con 8 torneos internacionales de los títulos más relevantes, como pueden ser Dota, League Of Legends, Starcraft, Hearthstone etc. También promueven torneos a nivel nacional, como el Red Bull Campus League de Rocket League el pasado 2017, un torneo en el que Red Bull se fue moviendo por todas las universidades españolas para encontrar al mejor jugador universitario de Rocket League, formando finalmente dos equipos que se batirían en un evento presencial en Gamepolis, Málaga. El segundo paso del marketing de contenidos de Red Bull en esta industria fue reclutar jugadores para sponsorizarlos como sus atletas, como hace en otras múltiples disciplinas del deporte tradicional. Algunos de los fichajes más destacados de estos patrocinios fueron Soren 'Bjergsen' Berg (League Of Legends), Enrique 'xPeke' Cedeño (League of Legends) y Mat 'NaDeSHoT' Haag (Call Of Duty). Su tercer paso fue patrocinar a organizaciones competitivas de esports, entre las que se encontraban los referentes americanos Team Solo Mid y Cloud 9 durante el 2016. A principios de 2017, con la creación del nuevo equipo de League Of Legends de Red Bull, el Red Bulls LoL Team, se tomó la decisión de no renovar los contratos de patrocinio con las dos organizaciones americanas para centrarse en su equipo propio de League Of Legends, pero tras el deportivo, el equipo se disolvió. Finalmente, para el 2018 volvió a entrar como patrocinador principal en Cloud 9, con un contrato mucho más fuerte y más involucrado en contenidos que el de hace dos años. Para esa fecha, Team Solo Mid ya había iniciado un nuevo patrocinador con Dr. Pepper, por lo que Red Bull no puedo volver a entrar en la organización. Finalmente, la última estrategia de marketing de contenidos de Red Bull ha sido crear series en video de alta calidad relatando la vida de estos jugadores de élite en las máximas competiciones de cada título.

- T Mobile: Una de las empresas de telecomunicaciones más grandes de Estados Unidos entró en los esports patrocinando a equipos americanos de las dos ligas más importantes de la región: La NA LCS, patrocinando a Cloud 9 y a 
Team Solo Mid desde agosto de 2017, y la OWL (Overwatch League) siendo el principal patrocinador de Houston Outlaw.

Otro fenómeno que recientemente está surgiendo a nivel internacional en los esports es la inclusión de marcas de lujo, como Louis Vuitton.

Según Graham Ashton, periodista para The Esports Observer, Louis Vuitton ha firmado un contrato de varios años con Riot Games para patrocinar los Worlds de League Of Legends, empezando por los de este 2019. El patrocinio incluirá tanto contenido físico como digital.

Dentro de lo que será el contenido físico de este patrocinio, Louis Vuitton diseñará un estuche dónde se almacenará la copa del mundo para transportarla hasta París, destino a donde se jugará la final.

Además, dentro del contenido digital, se creará un proyecto especial de pago para un personaje dentro del juego, el cual llevará ropa de Luis Vuitton y recibirá el nombre de la marca (Ashton, 2019).

Tras mencionar estos ejemplos internacionales, debemos comentar también cómo ha evolucionado la industria en España. Un caso de relevancia es el de las tres empresas de telecomunicaciones más grandes del país, Jaume Esteve resume cómo han entrado Vodafone, Orange y Movistar en el panorama español de los esports:

- Vodafone: Esta empresa británica fue la primera en entrar en la industria. Anunciaron su llegada en abril del 2016 con un nuevo equipo de League of Legends: G2 Vodafone, subsidiario en España de G2, equipo de LCS fundado por el exjugador español Carlos Rodríguez 'Ocelote'. Con la llegada de este equipo se lanzó algo nunca visto en los esports, una serie de MTV llamada Gamers que contó el día a día de aquel equipo (Fernández, 2017). Tras patrocinar a G2 en España durante más de año y medio, finalmente G2 decidió cambiar de proyecto, separando sus caminos con G2 y firmando un nuevo contrato para patrocinar a Giants, un histórico. Según afirma Borja Menotti, senior brand mánager de Vodafone en una entrevista a Expansión: "Nuestra ambición es convertir a Vodafone Giants en el mejor equipo profesional de deportes electrónicos del país" (Expansión, 2018)

- Movistar: El operador entró en los esports de la mano de la ESL, integrando un nuevo canal en su catálogo exclusivamente de esports, donde se producirían las competiciones de la ESL. Aparte del canal, que también crearía contenido propio de esports y videojuegos (Esteve, 2016). Paralelamente, Movistar lanzó su proyecto deportivo, su equipo Movistar Riders. Este tiene actualmente 8 equipos activos, entre los que destacan su equipo de League of Legends, que 
estuvo muy cerca de entrar en la máxima competición europea y su equipo de Counter Strike, que fichó en junio de 2017 al jugador español más icónico de todos los tiempos, Oscar Mixwell Canellas.

- Orange: A diferencia de sus otros dos rivales, no entró patrocinando ni formando equipos, lo hizo de una forma totalmente diferente y visiblemente dominante. Orange entró en los esports patrocinando a la propia Liga de Videojuegos Profesional, LVP, dándole nombre a la nueva liga española de máximo nivel en los títulos más importantes, la actualmente llamada Super Liga Orange.

Cabe mencionar que estas tres empresas se han sumado al marketing de contenidos, teniendo las tres un canal propio donde suben noticias, speedruns (un solo jugador), y tertulias sobre deportes electrónicos.

A estas tres compañías de telecomunicaciones, les han seguido otras marcas relevantes a nivel español en 2019, que también se han incorporado a los esports. Según Antevenio, un medio digital especializado en marketing de deportes electrónicos, las marcas más importantes que han incursionado a nivel nacional en los esports este 2019 han sido (Antevenio, 2019):

- Banco Santander: Este banco español entró en el año 2019 a patrocinar el torneo virtual LaLiga Esports, que es organizado por La Liga, la LVP, y EA.

- Font Vella: Esta marca de agua embotellada propiedad de Danone ha entrado en los esports a nivel nacional patrocinando a la LVP (Liga de Videojuegos Profesiona) y asociándose con cuatro de los clubes más importantes del panorama: Mad Lions, X6tence, Team Queso, y Giants. La acción inicial de este patrocinio fue sacar cuatro ediciones especiales de sus botellas de $50 \mathrm{CL}$ con los escudos de los respectivos equipos mencionados arriba. Además, estas botellas escondían un código en su etiqueta que daba acceso a participar en diferentes sorteos.

- Mapfre: La entrada de esta aseguradora ha sido una de las más relevantes este año 2019 dentro del panorama nacional. Primeramente, entraron como principal patrocinador de ESL Racing Series, competición de conducción a nivel internacional que permitirá relacionar a la marca con su actividad profesional real.

Además de esto, Mapfre patrocina a la vez a la competencia directa de ESL, la LVP, apoyando a los torneos de la Super Liga Orange en League Of Legends y Clash Royale. Por último, la aseguradora está explorando también las organizaciones que constituyen el futuro de los esports al patrocinar a GGTech, encargado de los 
principales torneos de deportes electrónicos dentro de los centros educativos con IEsports (centros educativos) y UniversityEsports (universidades).

Con relación a todas las empresas que hemos visto antes, según Robert Gotarra (2017) hay un esquema muy definido de cómo se posicionan para publicitarse en medio de una retransmisión de esports:

- Antes de que empiece la competición, hay una cortinilla con una vuelta atrás, donde se insertan los logos de las empresas patrocinadoras del evento.

- Antes del partido en sí, hay una mesa de análisis donde los comentaristas analizan previamente la jornada que se va a disputar, y en esas mismas mesas aparecen los logos de patrocinadores.

- Cuando los jugadores entran al escenario, las empresas colocan sus logos detrás de ellos para que sean totalmente visibles en ese plano.

- Una vez se sientan los jugadores, detrás de sus sillas aparecen paneles con logos, con mucha similitud a las vallas publicitarias de los deportes tradicionales.

- En la propia fase de selección de personajes prepartida, llamado 'Draft' aparecen en la viñeta los logos de las marcas perfectamente acoplados y en sintonía con el juego.

- Finalmente, cuando empieza la partida, los logos se integran en la interfaz del juego, de una manera que no molesta al consumidor al ver el partido. (Gotarra, 2017).

Por otro lado, tenemos las inversiones en marketing y el propio marketing de los clubs y organizaciones de esports. Partimos desde el punto que las cifras de cada entidad son privadas y variantes dependiendo de la organización por lo que solo se analizará las estrategias que siguen a la hora de hacer marketing.

No hay mucha información existente de cómo trabajan el marketing las organizaciones de esports, pero podemos ver claramente que es muy similar al marketing que utilizan los clubs y organizaciones de deporte tradicional. De hecho, muchos estos tienen ya su parte de deportes electrónicos, como puede ser el Valencia C.F. o el ThunderX3 Baskonia, la división de esports del Grupo Baskonia-Alavés y primer club deportivo en entrar de lleno en nuestro país. También, clubes como Schalke 04, Paris SaintGermain y FC Copenhague tienen sus divisiones en los esports (Guiñón Moliner, 2018). 
La estrategia que seguir a la hora de crear una base sólida de seguidores, hacer crecer su imagen y ganar reputación consiste principalmente en estrategias que siguen los clubes tradicionales y marketing de contenido. Algunas de estas estrategias están altamente relacionadas con los propios integrantes de la organización o del club $y$, normalmente, con los propios jugadores.

\section{METODOLOGIA}

La metodología de este estudio se basa en dos partes:

Primero se realiza un estudio de fuentes secundarias que pronostiquen la futura evolución de los esports en los próximos años, tanto económicamente como en visualizaciones. Este será un análisis de contenido que, como afirma Raigada, es:

Se suele llamar análisis de contenido al conjunto de procedimientos interpretativos de productos comunicativos (mensajes, textos o discursos) que proceden de procesos singulares de comunicación previamente registrados, y que, basados en técnicas de medida, a veces cuantitativas (estadísticas basadas en el recuento de unidades), a veces cualitativas (lógicas basadas en la combinación de categorías) tienen por objeto elaborar y procesar datos relevantes sobre las condiciones mismas en que se han producido aquellos textos, o sobre las condiciones que puedan darse para su empleo posterior (Raigada, 2002).

El principal estudio que analizar será Newzoo. Esta institución realiza anualmente un estudio de cómo avanza la industria, en el que también aparecen diversas predicciones para los próximos años. Además de este estudio anual, se recopilarán también los datos de un artículo adicional de Newzoo en el que se realizan 10 predicciones de la industria de los esports para 2021.

Tras este análisis de fuentes secundarias, se realizará una entrevista cualitativa. Según Vargas-Jiménez:

La entrevista cualitativa permite la recopilación de información detallada en vista de que la persona que informa comparte oralmente con el investigador aquello concerniente a un tema específico o evento acaecido en su vida". La misma continúa practicándose mano a mano con el método de la observación participante, aunque también esto es asumido por científicos cuantitativos a quienes les preocupan el rigor de la medición en investigaciones de grandes extensiones (Vargas-Jiménez, 2012).

En nuestras entrevistas se realizan las siguientes preguntas en relación con el sector estudiado: 
$1^{\circ}$ ¿Qué relación tiene con el sector? ¿Desde cuándo?

$2^{\circ}$ ¿Qué cambios destacaría desde que conoció la industria? ¿En qué factores ha aumentado el grado de profesionalización?

$3^{\circ}$ ¿Por qué el sector es atractivo para las marcas?

$4^{\circ}$ ¿Hay hueco para más títulos en los esports? ¿Se podría producir una burbuja por exceso de estos?

$5^{\circ}$ ¿Cómo evolucionará la industria? ¿Seguirá creciendo al mismo ritmo?

La entrevista ha sido realizada a los siguientes profesionales del sector:

- Bernando Bouzas: Social media mánager y encargado de comunicación y prensa de MAD LIONS EC.

- Sergio García Moreno Ramírez: Redactor en The Gaming House.

- Fernando Cardenete Aspiroz: Responsable de contenidos GGTech Entertainment.

- Ulises Cárcamo Fahnert: esports Lobbyist LATAM markets and Germany.

- Lucas Rojo Picó: Manager de la sección de PC en Team Queso.

\section{RESULTADOS}

Se han recopilado datos de Newzoo, la empresa más fiable de análisis de datos de lo esports actualmente, y esta estima que de cara a 2021, se estiman que las personas consumiendo serán más de 512 millones y que las ganancias serán de 3.035 millones de dólares para la misma fecha.

Newzoo, localizada en Holanda, es una de las empresas líderes de análisis de datos en videojuegos y deportes electrónicos. Ésta, en su último informe anual sobre esports (2018), estimó diferentes cifras en relación con el sector para los años venideros.

El pasado 2018, los esports tuvieron una audiencia de 380 millones de personas. Según las estimaciones de Newzoo, habrá un total de 512 millones de personas consumiendo deportes electrónicos en 2021, lo que significaría un aumento del $14,4 \%$ con respecto al 2018. En cuanto a facturación, el sector produjo un total de 1600 millones de dólares el pasado 2018. De nuevo, las estimaciones prevén un aumento de la facturación, llegando a un total de 3.035 millones de dólares de beneficios en 2021, lo que supondría un crecimiento del $27.4 \%$ con respecto al 2018. 
Tras estas predicciones de Newzoo, tenemos que sumar las conclusiones sacadas a partir de las respuestas de nuestros entrevistados. A pesar de que todos los entrevistados mencionan que es muy difícil predecir cómo evolucionará el sector (debido a su dependencia de la tecnología y su estructura cambiante), concluyen en que lo más probable es que siga creciendo, ya lo afirmen de manera más o menos optimista. Se habla de que hay una clara fiabilidad en un sector que cada vez está más formado y es más sostenible, pero que es muy dependiente de las marcas y los patrocinadores que quieran apostar a largo plazo por el sector (ya que a corto plazo no obtendrán el retorno de la inversión) y ayudarlo a superar esos retos que se le puede plantear.

Por otro lado, cabe destacar que todos nuestros entrevistados afirman que la profesionalización ha sido bastante notable. Fernando Cardenete, por ejemplo, añade el concepto de que un año de esports son como "un año de perro", por lo que se deduce que la evolución que se experimenta en un año en los deportes electrónicos suele ser exponencial comparado a otras industrias, como la del deporte tradicional. También coinciden en que el salto de profesionalidad fue notable una vez que entraron los contratos, los agentes, y las grandes marcas a los equipos profesionales, que hizo que los mejores jugadores dejaran de jugar por "hobby" a ser profesionales remunerados.

Según estos, los esports son un sector bastante atractivo para las marcas, por diversas razones. Aitorek afirma que el segmento de edad de los consumidores de esports es muy amplio, sin embargo, según estudios de Newzoo y palabras de Fernando Cardenete y Bernardo Bouzas, el segmento de edad de los esports se encuentra entre 14 y 28 años, de manera que las marcas pueden llegar a un público objetivo al que le costaría llegar de otra manera ya que no consumen deportes y medios tradicionales.

Por otro lado, hablando respecto a si puede haber o no una burbuja en lo deportes electrónicos, la mayoría confían en que no la habrá. Fernando Cardenete argumenta que posiblemente pase algo parecido al caso del fútbol en los deportes tradicionales, que mueve mucho más que otros deportes, habiendo esports que muevan a muchas más personas que otros. Ulises Cárcamo alega que el hueco que hay es ilimitado y que todo el que entre será regulado por la demanda de los espectadores. Sergio Moreno-García añade un nuevo factor a la cuestión y es que solo los deportes electrónicos que crecen por el interés de la propia comunidad de jugadores seguirán creciendo y se estabilizarán, mientras que otros esports que suben gracias a las grandes inversiones de sus desarrolladoras vayan rotando.

Por otro lado, se puede ver a continuación una tabla resumen del resultado de las entrevistas realizadas a modo de recopilación, donde se intenta mantener la idea del entrevistado de la forma más simple posible. 
Tabla 1. Resultado de las entrevistas realizadas

\begin{tabular}{|c|c|c|c|c|c|c|}
\hline & $\begin{array}{l}\text { Lucas } \\
\text { Rojo }\end{array}$ & $\begin{array}{l}\text { Bernardo } \\
\text { Bouzas }\end{array}$ & $\begin{array}{l}\text { Fernando } \\
\text { Cardenete }\end{array}$ & $\begin{array}{l}\text { Aitor Cru- } \\
\text { geira }\end{array}$ & $\begin{array}{l}\text { Sergio } \\
\text { Moreno- } \\
\text { Ramírez }\end{array}$ & $\begin{array}{l}\text { Ulises } \\
\text { Cárcamo }\end{array}$ \\
\hline $\begin{array}{l}\text { Relación } \\
\text { con el } \\
\text { sector }\end{array}$ & $\begin{array}{l}\text { Entrenador } \\
\text { de fortnite }\end{array}$ & $\begin{array}{l}\text { Social } \\
\text { Media } \\
\text { Manager } \\
\text { en Mad } \\
\text { Lions }\end{array}$ & $\begin{array}{l}\text { Periodista } \\
\text { especializa- } \\
\text { do en depor- } \\
\text { tes electróni- } \\
\text { cos }\end{array}$ & $\begin{array}{l}\text { Árbitro de la } \\
\text { Liga de Vi- } \\
\text { deojuegos } \\
\text { Profesional }\end{array}$ & $\begin{array}{l}\text { Redactor y } \\
\text { narrador de } \\
\text { competiciones }\end{array}$ & $\begin{array}{l}\text { Lobbista } \\
\text { especiali- } \\
\text { zado en } \\
\text { esports }\end{array}$ \\
\hline $\begin{array}{l}\text { Profesio- } \\
\text { nalización } \\
\text { de la in- } \\
\text { dustria }\end{array}$ & Creciente & Creciente & Creciente & Creciente & Creciente & Creciente \\
\hline $\begin{array}{l}\text { Atractivo } \\
\text { para las } \\
\text { marcas }\end{array}$ & $\begin{array}{l}\text { Atractivo } \\
\text { por la edad } \\
\text { del target }\end{array}$ & $\begin{array}{l}\text { Atractivo } \\
\text { por la } \\
\text { edad del } \\
\text { target }\end{array}$ & $\begin{array}{l}\text { Atractivo por } \\
\text { la edad del } \\
\text { target y por- } \\
\text { que no con- } \\
\text { sumen me- } \\
\text { dios tradicio- } \\
\text { nales }\end{array}$ & $\begin{array}{l}\text { Atractivo } \\
\text { porque el } \\
\text { target tiene } \\
\text { un segmento } \\
\text { de edad muy } \\
\text { amplio }\end{array}$ & $\begin{array}{l}\text { Atractivo por- } \\
\text { que llega a un } \\
\text { target difícil de } \\
\text { alcanzar }\end{array}$ & $\begin{array}{l}\text { Atractivo } \\
\text { por diver- } \\
\text { sos facto- } \\
\text { res }\end{array}$ \\
\hline $\begin{array}{l}\text { Posible } \\
\text { burbuja } \\
\text { por exce- } \\
\text { so de } \\
\text { títulos }\end{array}$ & No & & $\begin{array}{l}\text { Hay hueco } \\
\text { para más, } \\
\text { pero con } \\
\text { matices. }\end{array}$ & No & $\begin{array}{l}\text { Depende de si } \\
\text { crece por el } \\
\text { apoyo de su } \\
\text { comunidad o } \\
\text { por una fuerte } \\
\text { inversión }\end{array}$ & No \\
\hline $\begin{array}{l}\text { Evolución } \\
\text { en el futu- } \\
\text { ro }\end{array}$ & $\begin{array}{l}\text { Crecerá, } \\
\text { pero se } \\
\text { autorregu- } \\
\text { lará }\end{array}$ & $\begin{array}{l}\text { Crecerá } \\
\text { exponen- } \\
\text { cialmente }\end{array}$ & $\begin{array}{l}\text { Depende de } \\
\text { cómo afronte } \\
\text { las principa- } \\
\text { les incógni- } \\
\text { tas }\end{array}$ & $\begin{array}{l}\text { Todo apunta } \\
\text { a que crece- } \\
\text { rá, pero } \\
\text { depende de } \\
\text { la inversión } \\
\text { de las mar- } \\
\text { cas }\end{array}$ & $\begin{array}{l}\text { Crecerá, pero } \\
\text { existe la posi- } \\
\text { bilidad de que } \\
\text { se desinfle }\end{array}$ & $\begin{array}{l}\text { Crecerá } \\
\text { exponen- } \\
\text { cialmente }\end{array}$ \\
\hline
\end{tabular}

Fuente: Elaboración propia 


\section{CONCLUSIONES}

Tras analizar estos últimos 5 años de los esports, donde hemos visto que ha habido un crecimiento exponencial de la industria, se ha podido sacar unas conclusiones bastantes lógicas de ellos, y es que a priori, se puede esperar una continuación del crecimiento para los próximos años, sin recurrir a ningún otro dato.

Para hacer estas conclusiones más sólidas, se han cogido los datos de Newzoo relativos a las predicciones para el año 2021, que como se esperaban, son datos que confirman lo que ya se puede suponer analizando los datos de 2013-2019, que es un crecimiento de la industria en números y dinero.

Por último, se han tomado datos de personas expertas en el sector para ver sus análisis personales de la industria desde dentro y como profesionales que llevan años trabajando en ella. De una forma u otra, todos están en sintonía con que la industria seguirá creciendo durante los próximos años.

Con todos estos datos, que confirman nuestra hipótesis inicial de que la industria de los esports está creciendo, y seguirá creciendo durante los siguientes años mínimo, podemos asegurar que, de forma directa, la inversión en marketing que se ejercerá en el sector evolucionará conforme a ella.

Tras la entrada de diferentes empresas grandes en la industria a nivel nacional e internacional para hacer marketing (como son los casos anteriormente expuestos de Movistar, Red Bull, Coca-Cola, Intel, etc.), los datos ya analizados, los pronósticos para los próximos años y la opinión de la gente relacionada con el sector, se puede confirmar que dada a la profesionalización del mismo, las empresas ya pueden entrar en el negocio con proyectos que tengan una seguridad de continuidad a medio-largo plazo, aunque aún no se garantice un retorno de la inversión.

Como dicen las estimaciones del capital que moverá la industria en 2021, no solo las empresas ya presentes, si no que nuevas empresas entrarán al sector en busca de hacer marketing en una industria creciente y muy interesante, creando a su vez un mayor presupuesto para clubes $u$ organizaciones, o incluso la formación de nuevas empresas relacionadas con el negocio.

Asimismo, los clubes o las organizaciones del sector dispondrán, debido a lo mencionado anteriormente, de un mayor presupuesto. Ya sea por inversión propia o de empresas y marcas externas, podrán desarrollar sus departamentos, entre los que se incluye el departamento de marketing y todo lo relacionado a este. Cada organización tiene estrategias diferentes, pero se podrá ver de forma general un mayor marketing de contenidos y diferentes estrategias de marketing de las diferentes organizaciones, como ya realizan los clubes tradicionales de deportes en su búsqueda de nuevos "seguidores". 


\section{Referencias}

AEVI (2018). Libro blanco de los esports en España. Recuperado de https://bit.ly/2QOoz4I

Ashton, G. (2019). Louis Vuitton to Design Trophy Case for League of Legends World Championships. Recuperado de https://bit.ly/2WLRUQC

Baker, C., \& Baker, C. (2016). Stewart Brand Recalls First 'Spacewar' Video Game Tournament - Rolling Stone. Recuperado de https://bit.ly/3bp3tB|

Bulls, R. (2018). Red Bulls. Recuperado de https://bit.ly/2Ui4Z2w

Cambridge (2018). Definición de esports en el Diccionario Cambridge inglés. Recuperado de https://bit.ly/2JITBfv

Campoy, D. (2018). Claves para que una marca entre en el sector de los esports (con éxito). Recuperado de https://bit.ly/2R20ZkP

Coke esports (2018). The Coca-Cola Company. Recuperado de https://bit.ly/2Un6kVT

Communique of the Olympic Summit. (2017). Recuperado de https://bit.ly/2Uh55r8

Esports history and evolution.(2018). Recuperado de https://bit.ly/2WHWteM

Esteve, J. (2016). Las operadoras se lanzan al negocio de los esports (y puede ser un gran error). El Confidencial. Recuperado de https://bit.ly/2QLU9j6

Expansión (2018). Vodafone se alía con el equipo español de esports Giants, Expansión, 7 de abril de 2018. Recuperado de https://bit.ly/2UkN1MY

Gaudiosi, J. (2014). Call Of Duty tops Western esports audience. Recuperado de https://win.gs/2wGNUX1

Gotarra, R. (2017). Patrocinio en los esports. Recuperado de https://bit.ly/2wGAj1I

Guiñón Moliner, A.M. (2017). Los clubes de fútbol europeos con presencia en los esports. Recuperado de https://bit.ly/3awitgV

Inside Esports. (2017). Marcas y patrocinios en esports: un análisis de su desarrollo en 2017. Recuperado de https://bit.ly/2vQCShx

Intel en los deportes electrónicos: Impulsando a los líderes de los... (2018). Recuperado de https://intel.ly/2wtTMmv

Kresse, C. (2016). Red Bull esports: King of Content Marketing. Recuperado de https://bit.ly/3doutme

Lazzari, L (2017). A Comprehensive History of esports. Recuperado de https://bit.ly/3ao08SQ

El Economista (2018). Los esports ya son parte de la estrategia de marketing y patrocinio de las empresas españolas.Recuperado de https://bit.ly/2xopMs7

Marco, Á. (2018). Esports: ¿qué deben saber las marcas?. Recuperado de https://bit.ly/2QPj718

Martin, R. (2017) Juegos MOBA con los que escapar del monopolio de League of Legends, Recuperado de EI Diario, 7/08/2017 https://bit.ly/39jQaR9

Mediavilla, J. (2018). ¿Cómo debe ser el club de esports ideal? En esports as. Recuperado de: https://bit.ly/2vPa09g 
Meola, A. (2018). The biggest companies sponsoring esports teams and tournaments. Recuperado de https://bit.ly/2UFkjoD

Montero, E. (2015).Los esports superan a la NBA - MARCA.com. Recuperado de https://bit.ly/2Q010xc

Murray, T. (2018). Houston Outlaws Announce Partnership with T-Mobile - The esports Observer. Recuperado de https://bit.ly/2wGOrlv

Newzoo. (2016). Global esports Market Report: Revenues to Jump to $\$ 463 \mathrm{M}$ in 2016 as US Leads the Way. Recuperado de https://bit.ly/2QLIbGa

TSM Site. (2018). Recuperado de https://tsm.gg/partners

Antevenio 2018. Principales patrocinadores de esports en España. Recuperado de https://bit.ly/33OhTZm

Raigada, J. L. P. (2002). Epistemología, metodología y técnicas del análisis de contenido. Sociolinguistic Studies, 3(1), 1-42.

Synnaeve, G., \& Bessiere, P. (2012). A dataset for StarCraft Al and an example of armies clustering. In Eighth Artificial Intelligence and Interactive Digital Entertainment Conference.

The History and Evolution of esports - Bountie Gaming - Medium. (2018). Recuperado de https://bit.ly/3bqDauv

Vargas-Jiménez, I. (2012). La entrevista en investigación cualitativa: nuevas tendencias y retos. Revista Calidad en la Educación Superior. Calidad en la Educación Superior, 1, 119139 -

Wagner, M. G. (2006). On the Scientific Relevance of esports. In International conference on internet computing (pp. 437-442).

Warman, P. (2017). Esports revenues will reach \$696M in 2017 and $\$ 1.5 \mathrm{Bn}$ by 2020. Recuperado de https://bit.ly/2UvKuOY

Warr, P. (2014). Esports in numbers: Five mind-blowing stats. Recuperado de https://win.gs/2xpVPla

Seo, Y. (2013). Electronic esports, a new marketing landscape of the experience economy. Recuperado de https://bit.ly/3dFmDVE

Yuste, A. (2016). Esports: El alcance de los deportes electrónicos en cifras. Recuperado de https://bit.ly/3bsr7wl 